\title{
Une tétrarhynchose vasculaire des thons du Golfe de Guinée due aux larves plerocercus de Dasyrhynchus talismani R. Ph. Dollfus 1935
}

\author{
par J. BUSSIERAS et J. F. ALDRIN \\ (Laboraloire de Parasıtologıe de l'Ecale Nationale Vétérinaire de Lyon, \\ Laboratoire de Contrôle des Produits de la Pêche, Abidjan, Côte d'lvoire)
}

\section{RÉSUMÉ}

Les thons Thunnus obesus Lowe et, à un moindre degré, Thunnus albocores Bonnaterre pêchés dans le Golfe de Guınée hébergent dans leur appareil circulatorre (vaisseaux du foie, de la rate et des caecums pyloriques) les larves plerocercus d'un Cestode de l'ordre des Tétrarhynques, Dasyrhynchus talismani R. Ph. Dollfus 1935.

Il est souvent difficıle d'étudier de façon systématique les infestations parasitaires des poissons marins ; c'est le cas notamment pour les infestations viscérales des gros Thunnidae tropicaux, le thon obèse ou patudo (Thunnus obesus Lowe, = Parathunnus obesus Lowe) ef l'albacore (Thunnus albacores Bonnaterre, $=$ Neothunnus abacoro Lowe). Ces poissons sont en effet fréquemment éviscérés et ébranchiés par les pêcheurs dès la sortie de l'eau.

Or ces espèces ont une grosse importance économique; à titre d'exemple, les captures des palangriers japonais d'avrıl 1961 à mars 1962, se sont réparties comme suit :

Thon albacore (T. albacores) ...... $142.000 \dagger$ Thon obèse (T. obesus) ..... . . . . $109.000 t$ Thon blanc ( $T$, alolungo) ......... $78.000 \dagger$ Thon rouge ( $T$. thynnus) $\ldots \ldots \ldots . .68 .000 \dagger$

De tels chiffres explıquent l'intérêt croissant porté à ces Thunnidae; c'est ainsi qu'en juın 1964, la Sous-Direction des Pêches de Côte-d'Ivoire a demandé aux quatre équipages japonaıs résidant à Abidjan, de ramener ces poissons non éviscérés, en vue de les étudier de façon plus complète.

Cela nous a permis de constater l'existence et l'extrême fréquence d'une infestation viscérale, à notre connaıssance non encore signalée, du patudo et de l'albacore par des larves de Tétrarhynques.

LOCALISATION DES PARASITES ET DESCRIPTION DES LÉSIONS.

1. Chez le thon obèse :

On est frappé, à l'examen des plexus vasculaires ef des vaisseaux sanguins de la région hépato-splénique, par l'existence de déformations des parois ef le développement d'anévrismes (fig. 1).

Si l'on incise ces parois vasculaires, de volumıneux vers cylindriques, blanchâtres, jaillissent au dehors et peuvent facilement être extirpés. La présence d'une dizaine de vers dans un même réseau vasculaire n'est pas exceptionnelle, et l'obstruction des valsseaux est souvent totale 


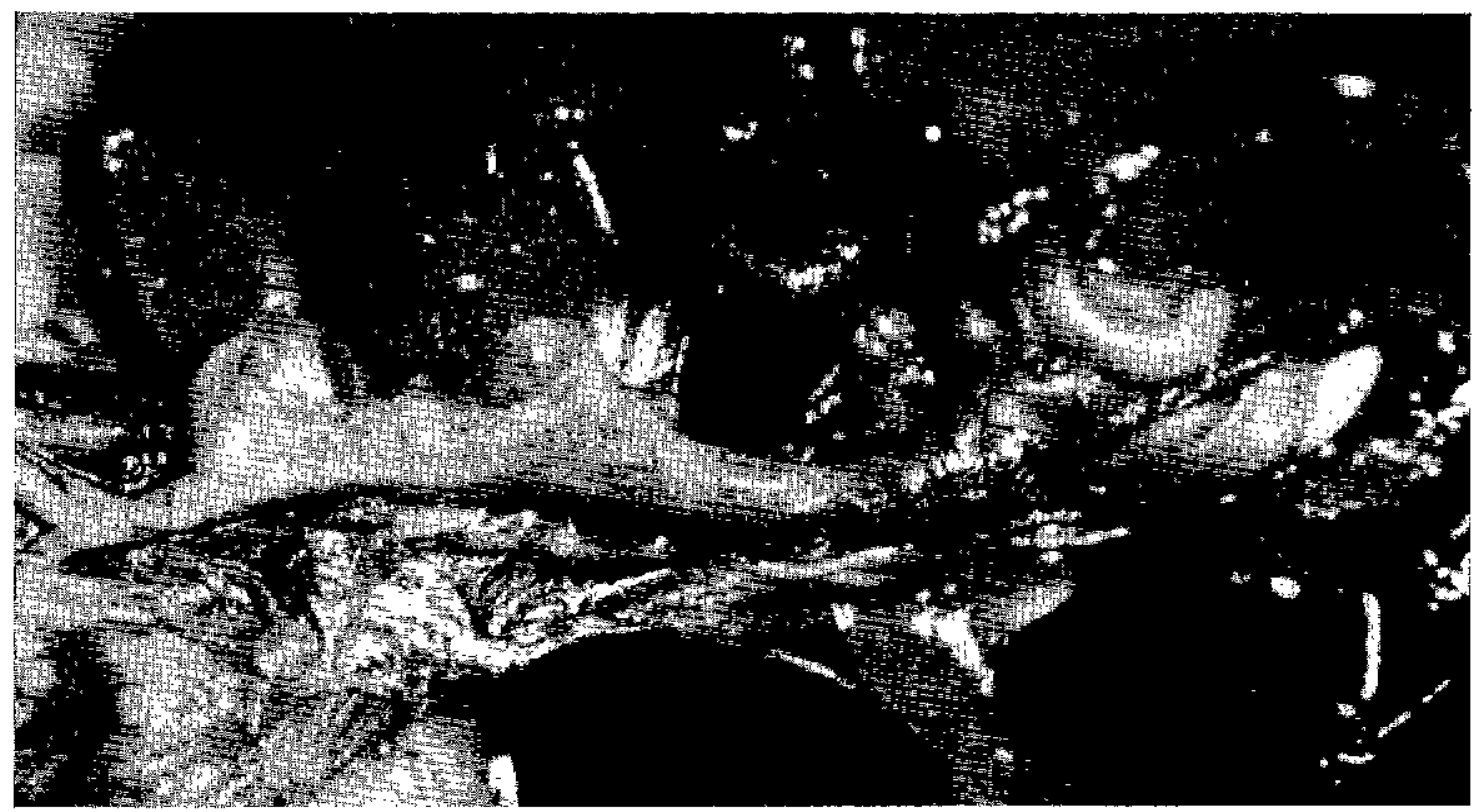

Fig. 1. - Lésions des vaisseaux hépatiques de Thunnus obesus, dues aux larves de Dasyrhynchus talismant (larves visıbles par transparence sur la partıe drolie du clıché).

II arrive que l'on trouve des vers dans la lumière des vaisseaux du foie, mais le parenchyme lui-même est toujours indemne, cinsı que la vésicule biliaire ef son canal excréteur. Par contre, les parasites pénètrent parfois dans le parenchyme splénique, qui est, il est vrai, en rapport étroit avec l'appareil circulatoire.

Les vaisseaux des caecums pyloriques sont parfois parasités eux aussi.

Enfin, nous n'avons jamais trouvé ces vers dans d'autres organes, ni dans d'autres vaisseaux (encore qu'une exploration complète de ces derniers soit difficile, voire impossible), ni même libres dans la cavité générale.

\section{Chez le thon albacore:}

Un examen systématıque d'un grand nombre de thons albacores a permis de retrouver chez eux le même parasitisme, mais beaucoup plus discret, parfois même difficile à déceler.

A la différence des patudos, les albacores présentent rarement des parasites dans les vaisseaux du foie; c'est surtout au niveau des vaisseaux des caecums pyloriques que l'on retrouve les vers, et généralement en petit nombre. Cette différence est peut-être à rapprocher du fait que chez Thunnus albacores le système vasculaire sous-hépatique est moins développé que chez le patudo.

\section{FRÉQUENCE DES INFESTATIONS.}

L'examen de 149 thons obèses, entre le 10 juın et le 31 décembre 1964, a montré que tous ces poissons, sans exception, étaient parasités. Les plus petits spécimens étudiés pesaient environ $5 \mathrm{~kg}$, et les plus gros avoisincient $100 \mathrm{~kg} ;$ d'une façon générale, les sujets âgés étaient plus lourdement infestés.

Depuis août 1964 jusqu'à février 1965, 505 albacores ont été examinés; sur ce total, les parasites ont été retrouvés chez 159, soit 31,5 p. 100. Chez les spécimens d'un poids inférieur à $10 \mathrm{~kg}$, le pourcentage est le plus faible: 17 sur 117 , soit environ 14,5 p. 100. Au contraire, les thons d'un poids supérieur d̀ $30 \mathrm{~kg}$ étaient presque toujours infestés : 18 sur un total de 21.

Ajoutons que 12 thons blancs, ou germons (T. alalunga) ont pu être étudiés: aucun d'eux n'était parasité. 


\section{DESCRIPTION DU PARASITE.}

L'étude parasitologıque montre qu'ıl s'agif de la même espèce de Cestodes Tétrarhynques (= Trypanorhyncha Diesing 1863) chez le patudo et chez l'albacore: Dosyrhynchus talisman R. Ph. Dollfus 1935 (fam. Dasyrhynchidae R. Ph. Dollfns 1935) ; l'étude extrêmement détaillée de Dollfus (publiée en 1942) concernait uniquement des spécimens adultes récoltés dans le tube digestif d'un requin bleu, Prionace glauca (= Carcharlas glaucus), capturé dans I'Atlantıque entre Dakar ef les lles du Cap Vert. Dans notre description, nous utiliserons d'alleurs la terminologie très claire et précise préconısée par Dollfus dans son travail.

1.' - La larve plerocercus complète (fig. 2) se présente comme un ver cylındroıde, blanchâtre, de 40 d̀ $120 \mathrm{~mm}$ de longueur et de 2 à $3 \mathrm{~mm}$ de diamètre ; I'une des extrémıtés forme un renflement olivaire, dont le grand axe mesure 8 à $10 \mathrm{~mm}$ et le petit axe 4 à $5 \mathrm{~mm}$. Si l'on incise la paroi à ce niveau, on trouve un long scolex invaginé, replié 3 ou 4 fois sur lui-même, et pourvu postérieurement d'un court oppendix : ce dernier est réuni à la partie externe de la larve (ou blastocyste) par un petit pédicule

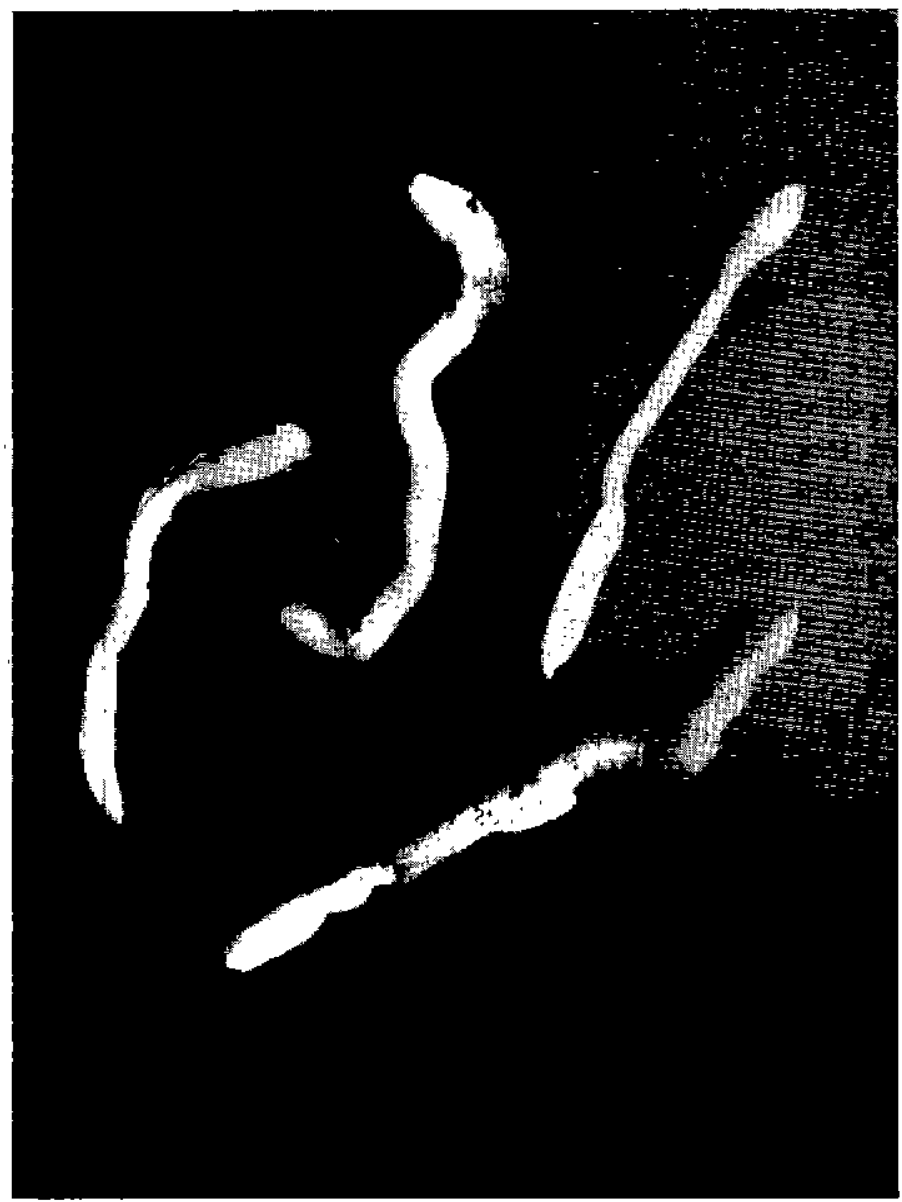

Fig. 2. - Larves de D. tolismani isolées $(\times 1,25)$. 
2. - L'ensemble scolex + appendix (fig. 3) a des dimensions assez constantes : longueur (trompes non comprises) $19 \mathrm{~mm}$, largeur (vers la mi-longueur) 1,9 à $2 \mathrm{~mm}$.

Les diverses parties de cet ensemble mesurent en moyenne:

Trompes

$2,2-2,4 \mathrm{~mm}$

Pars bothridialis $1,7-1,9 \mathrm{~mm}$

Pars vaginalis $7,4-8,6 \mathrm{~mm}$ (non compris p. bothr.)

Pars bulbosa ................. 6,8-7,3 $\mathrm{mm}$ Appendix

$2,2-2,9 \mathrm{~mm}$

Le scolex proprement dit ayant déjà été décrit de façon très détaillée dans le cas du parasite adulte, nous ne retiendrons que les éléments permettant la détermination de l'espèce :

a) Les trompes sont du type «pécilacanthe 》, caractérisé par la présence, sur le côté externe de la région métabasale, de crochets particulıers, en file longitudınale formant une «chaînette »: chez D. tolısmani, cette chaînette est très courte, constituée de 14 à 16 éléments seulement: chacun de ces éléments forme un écusson trapézoïdal, qui porte, vers le tiers de sa longueur, une pointe triangulaire (fig. 4).

Sur les deux faces, dites bothridiale et antibothridiale, de chaque trompe, partent du côté interne des rangées obliques de crochets principaux (fig. 5); chaque rangée se termine du côté externe, et couvre donc la moitié du tour de la trompe. A partir de la région de la chaînette, on remarque en outre la présence de petites rangées de crochets intercalaires, au nombre d'une rangée de crochets intercalaires pour une rangée de crochets principaux (fig. 4); cette disposition caractérise $D$. talismani, les autres espèces du genre Dasyrhynchus possédant au moins deux rangées de crochets intercalaires pour une de crochets principaux (de plus, dans ces autres espèces, la chaînette est formée d'éléments beaucoup plus nombreux).

Par ailleurs, en région basale, les trompes présentent, sur le côté externe, un ensemble de crochets très longs, formant une armature basale (fig. 5).

b) Les bothridies, au nombre de deux, ont la forme d'un coeur renversé, avec une échancrure postérieure et, sur la plupart des exemplaires. un bourrelet périphérique très marqué (fig. 6 et7).

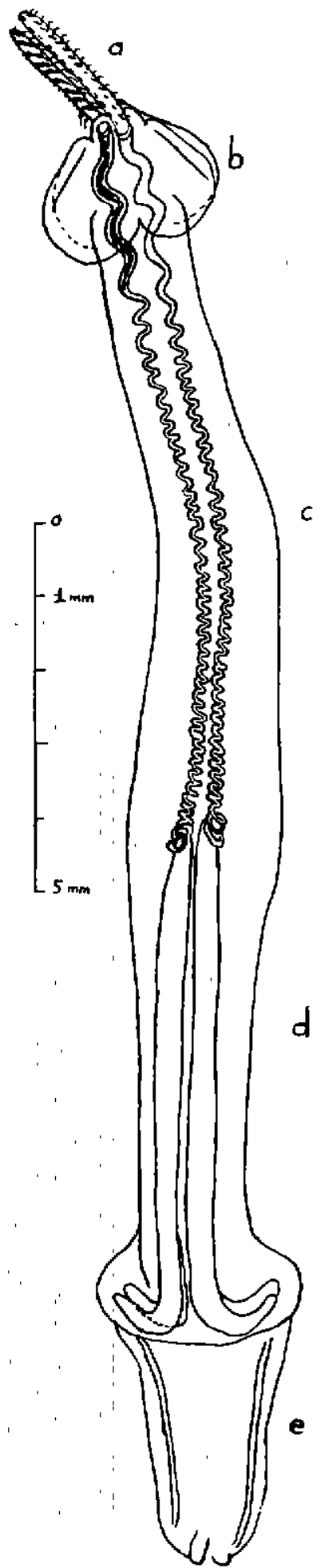

Fig. 3. - Larve de D. talismani : scolex et appendix a : trompes, b : pars bothridiolis, $c$ : pars vogmalis, $\mathrm{d}$ : pars buibosa, e : appendix. 


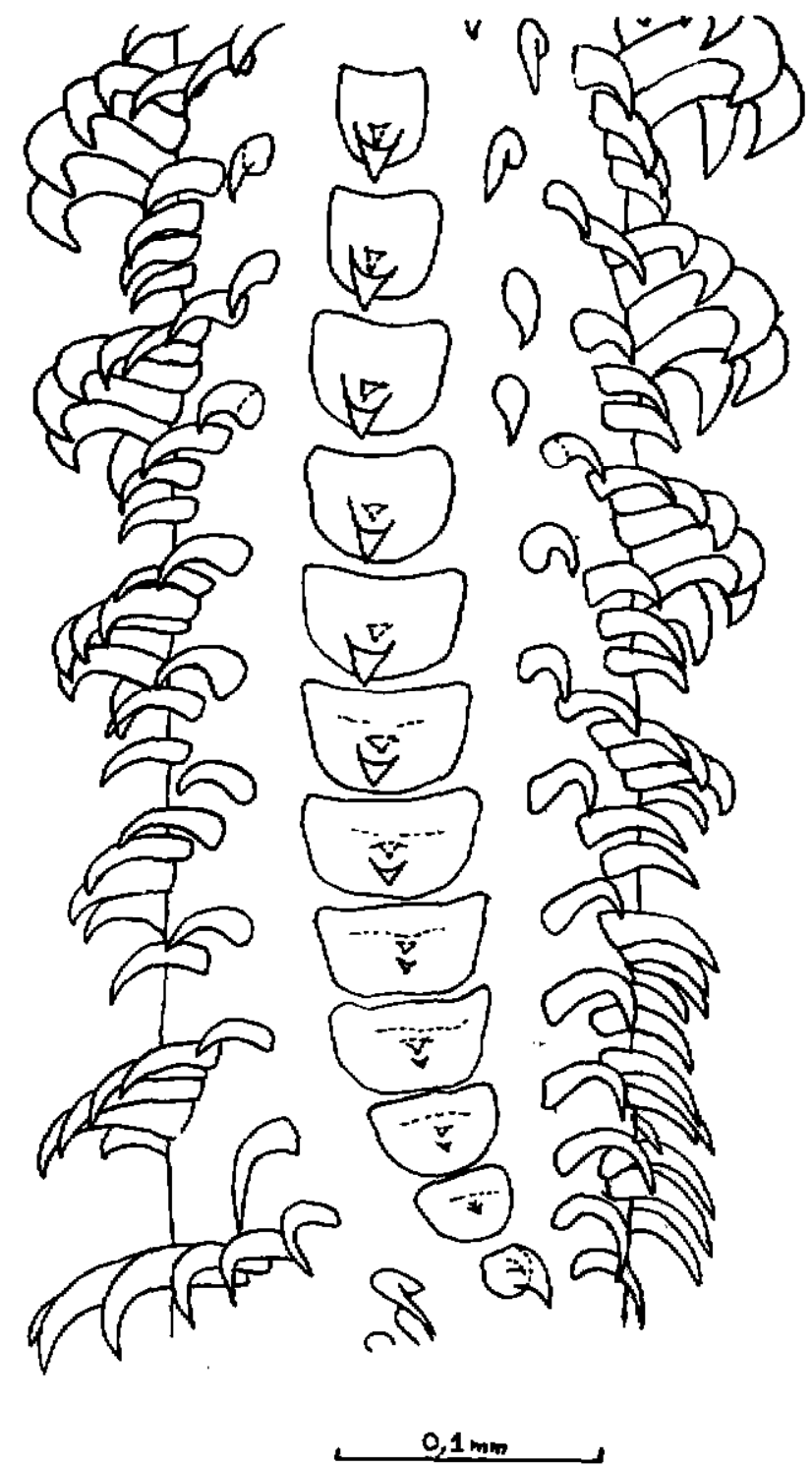

Fig. 4. - Partie métabasale d'une Irompe de $D$. talismoni, vue par son côté externe (la face bolhridiale est à gauche) : remarquer la chainelte, au centre, et de chaque côté l'alternance des rangées oblıques de crochets principaux et de crochets intercalaires (plus petits et moins nombreux).

La forme largement arrondie en avant observée par, DOLLFUS sur un exemplaire n'a pas été retrouvée.

c) La pars vaginalis renferme les 4 gaines vaginales fortement spiralées ; sur deux exemplaines s'observe très nettement une inversion du sens d'enroulement des spires, vers les $2 / 3$ de la longueur de cette pars vaginalis. d) Dans la pars bulbosa, les 4 bulbes musculaires sont, comme chez tous les Dasyrhynchus, extrêmement allongés ( 18 à 20 fois plus longs que larges), ef l'extrémité postérieure de chaque bulbe est incurvée vers l'extérieur (fig. 3).

La pars post-bulbosa est pratıquement absente, et, à la jonction avec l'appendix, on constate que le scalex est faiblement craspédote. 


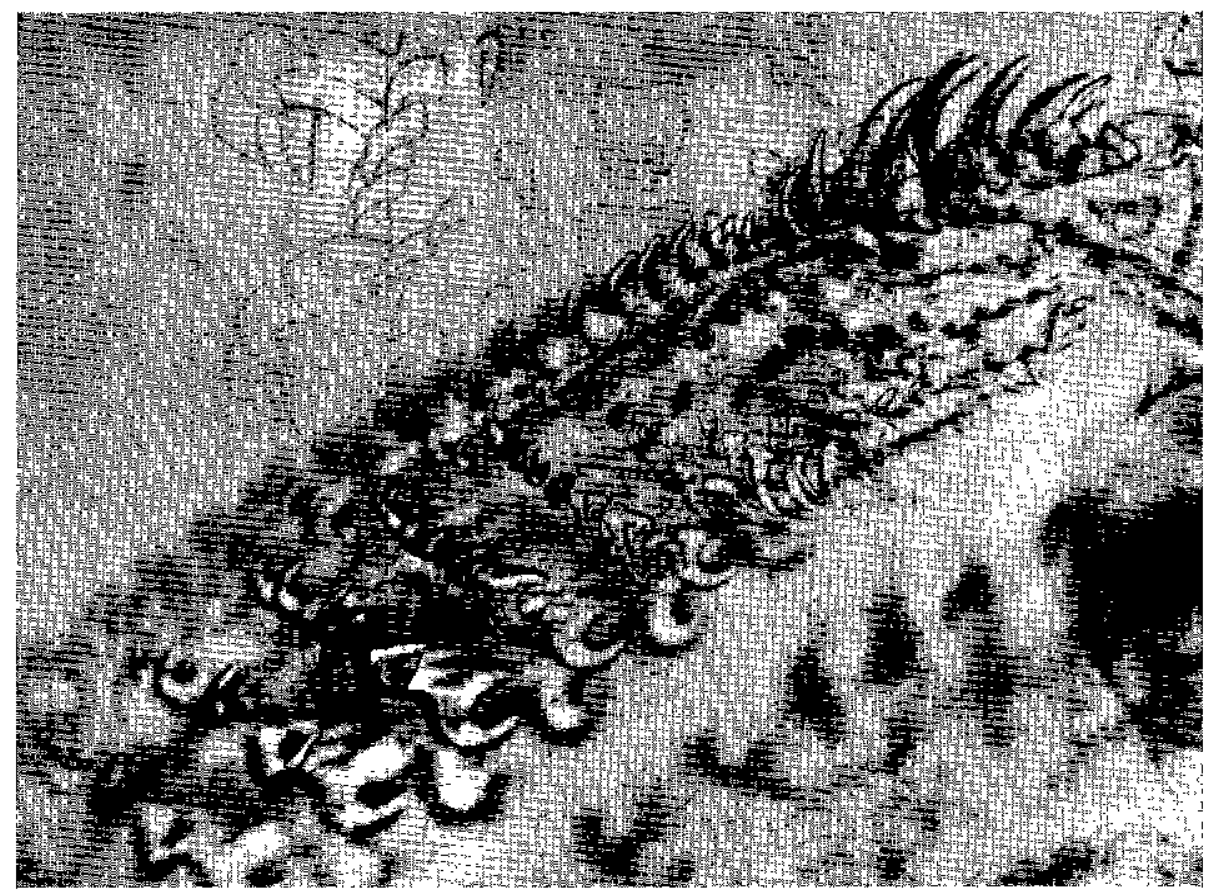

Fig. 5. - Parties basale et métabasale d'une trompe de D. tolismani vue par sa face antibothridiale $(x 145)$. Observer les rangées obliques de crochets principaux, et l'armature basale visible sur la photo en hauf et à droite.

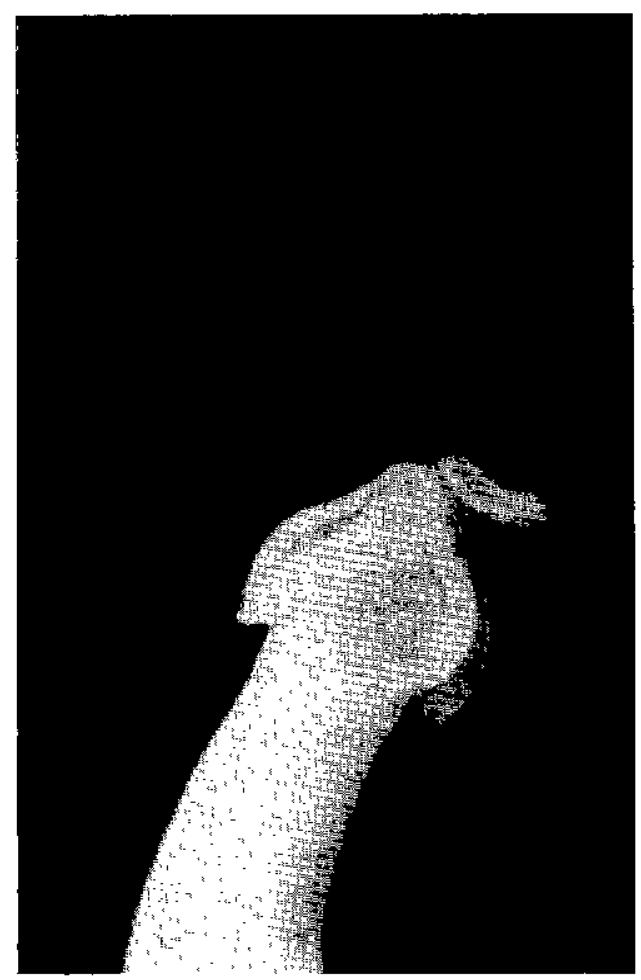

Fig. 6. - - Bothridies de D. tolismani, vues de face $(\times 12,5)$.

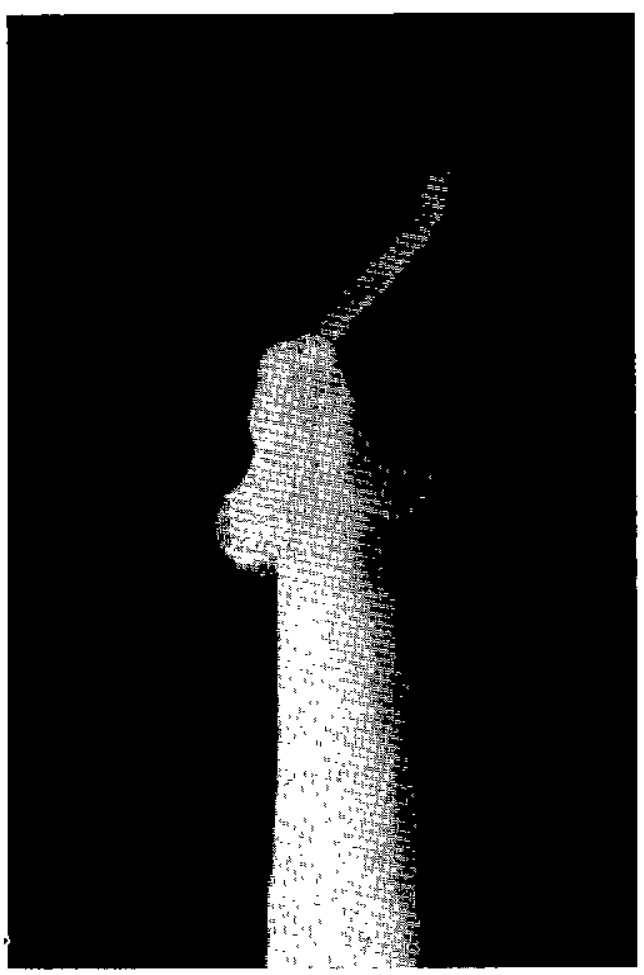

Fig. 7. - Bothridies de D. tolismani, vues de côté $(\times 12,5)$. 
e) L'oppendix, peu développé, contient des canaux excréteurs qui convergent postérieurement vers le point de jonction avec le blastocyste.

\section{DISCUSSION.}

1. - Il est surprenant de constater la fréquence et l'importance de l'infestation de $T$. obesus et T. albacores par les larves de Dasyrhynchus talismoni, ce qui rend difficilement compréhensible l'absence d'observations antérieures sur ce sujet.

Rappelons que l'éviscératıon habituellement subie par les thons au moment de la pêche fourn it une explication au moins partielie; mais nous sommes aussi amenés a supposer que les thons patudos et albacores des autres mers tropicales n'hébergent pas le parasıte, ce qui seraıt évidemment à vérifier.

2. - D'autre part, l'existence de Dasyrhynchus talismani adulte chez le requin bleu permet d'imaginer ce que peut être une partıe du cycle évolutif, les thons patudos et albacores intervenant comme seconds hôtes intermédiaires; Il est d'ailleurs souvent observé par les pêcheurs que les requins bleus viennent dévorer les thons déjà capturés sur les palangres.

\title{
SUMMARY
}

\begin{abstract}
A vascular Tetrarhynchosis of the tunnies of the Gulf of Guined due to the plerocercus larvae of Dosyrhynchus tolismani R. Ph. Dollfus 1935

The Thunnus obesus Lowe tunnies and, in the lower stage, the Thunnus albacores Bonnaterre, fished in the Gulf of Guinea harbour in their circulatory system (liver, spleen, pyloric blind-gut vessels) the plerocercus larvae of a cestode of Tetrarhynque order, Dasyrhynchus talismani R. Ph. DOLLFUS 1935.
\end{abstract}

\section{RESUMEN}

Una Tetrarincosis vascular de los atunes del Golfo de Guinea debida a las larvas plerocercus del Dasyrhynchus talismoni R. Ph. Dollfus 1935

Los atunes Thunnus obesus Lowe y a un menor grado. Thunnus albocores Bonnaterre pescados en el Golfo de Guinea albergan en su aparato circulatorio (vasos del higado, del bazo y de los cecos piloricos) las larvas plerocercus de un céstodo del orden de los Tetrarincos, Dasyrhynchus talisman R. Ph. DOLLFUS 1935.

\section{BIBLIOGRAPHIE}

ALDRIN (J. F.). - Observations sur la technologie ef l'inspection des Thonidés. Rec. Méd. Vét. (sous presse) 1965.

DOLLFUS (R. Ph.). - Sur quelques Tétrarhynques (Notes préliminaires). Bull. Soc. Zool. Fronce, 1935, 60 (3-4) : 353-7.

DOLLFUS (R. Ph.). - Etudes critiques sur les Tétrarhynques du Muséum de Paris. Arch. Mus. Nat. Hist. Nat., 1942, 6e série, 19, 1-466.

KISHINOUYE (K.). - Contribution to the compa- rative study of the so-called scombroid fishes. J. Coll. Agric., 1923, 8 (3).

LINTON (E.). - Notes on cestode parasites of sharks and skates. Proc. U. S. Nat. Mus, 1924, 64 (2511) : 1-114.

WARDLE (R. A.) et MCLEOD (J. A.). - The Zoology of Tapeworms. Univ. of Minnesota Press, 1952, 780.

YAMAGUTI (S.). - Systema helminthum. The Cestodes of Vertebrates. Interscience Publishers, New York. 1959, 2 : 860. 